\title{
Upper limb robotic rehabilitation for patients with cervical spi- nal cord injury: a comprehensive review
}

Giovanni Morone ${ }^{1 * *}$, Alessandro De Sire ${ }^{2 *^{*}}$, Alex Martino Cinnera ${ }^{1}$, Matteo Paci ${ }^{3}$, Luca Perrero ${ }^{4}$, Marco Invernizzi ${ }^{5,6}$, Lorenzo Lippi ${ }^{5}$, Michela Agostini ${ }^{7}$, Irene Aprile ${ }^{8}$, Emanuela Casanova ${ }^{9}$, Dario Marino ${ }^{10}$, Giuseppe La Rosa $^{11}$, Federica Bressi ${ }^{12}$, Silvia Sterzi ${ }^{12}$, Daniele Giansanti ${ }^{13}$, Alberto Battistini ${ }^{9}$, Sandra Miccinilli ${ }^{12}$, Serena Filoni ${ }^{14}$, Monica Sicari ${ }^{15}$, Salvatore Petrozzino ${ }^{15}$, Claudio Marcello Solaro ${ }^{16}$, Stefano Gargano ${ }^{17}$, Paolo Benanti ${ }^{18}$, Paolo Boldrini $^{19}$, Donatella Bonaiuti ${ }^{19}$, Enrico Castelli ${ }^{20}$, Francesco Draicchio ${ }^{21}$, Vincenzo Falabella ${ }^{22}$, Silvia Galeri ${ }^{23}$, Francesca Gimigliano ${ }^{24}$, Mauro Grigioni ${ }^{13}$, Stefano Mazzoleni ${ }^{25}$, Stefano Mazzon ${ }^{26}$, Franco Molteni ${ }^{27}$, Maurizio Petrarca $^{28}$, Alessandro Picelli ${ }^{29}$, Federico Posteraro ${ }^{30}$, Michele Senatore ${ }^{31}$, Giuseppe Turchetti ${ }^{32}$, and Sofia Straudi ${ }^{33}$, working group upper limb "CICERONE" Italian Consensus Conference on Robotic Rehabilitation

*These authors equally contributed

Collaborators:

Gandolfi Marialuisa ${ }^{29}$, Angela Palomba ${ }^{34}$, Chiara Arienti ${ }^{3}$, Ludovica Baluardo ${ }^{33}$, Emanuele Francesco Russo ${ }^{14}$, Manuela Desilvestri $^{6}$, Marco Bravi ${ }^{12}$, Diletta Bruno ${ }^{12}$, Fabio Santacaterina ${ }^{12}$, Cristiano Pecchioli ${ }^{35}$, Valentina Boetto ${ }^{36}$

1 Santa Lucia Foundation, IRCCS, Rome, Italy; m.tramontano@hsantalucia.it, g.morone@hsantalucia.it, a.martino@hsantalucia.it

2 Physical and Rehabilitative Medicine, Department of Medical and Surgical Sciences, University of Catanzaro "Magna Graecia", 88100 Catanzaro, Italy alessandro.desire@unicz.it

3 AUSL (Unique Sanitary Local Company) District of Central Tuscany, Florence, Italy. matteo.paci@applicazione.it,

4 Neurorehabilitation Unit, Azienda Ospedaliera SS. Antonio e Biagio e Cesare Arrigo, Alessandria, Italy, EU 4; lperrero@ospedale.al.it

5 Physical and Rehabilitative Medicine, Department of Health Sciences, University of Eastern Piedmont “A. Avogadro", 10121 Novara, Italy

6 Translational Medicine, Dipartimento Attività Integrate Ricerca e Innovazione (DAIRI), Azienda Ospedaliera Nazionale SS. Antonio e Biagio e Cesare Arrigo Alessandria, 15121 Alessandria, Italy

7 Department of Neuroscience, section of rehabilitation, Universi ty-General Hospital of Padova, Italy ; michela.agostini@unipd.it

8 IRCCS Fondazione Don Carlo Gnocchi, Florence, Italy; iaprile@dongnocchi.it

9 IRCCS Istituto delle Scienze Neurologiche di Bologna, UOC Medicina Riabilitativa e Neuroriabilitazione, Bologna, Italia 9; e.casanova@ausl.bologna.it; a.battistini@ausl.bologna.it.

10 IRCCS Neurolysis Center "Bonino Pulejo", Messina, Italy; dario.marino95@gmail.com

11 C.S.R. - Consorzio Siciliano di Riabilitazione, Catania, Italy; laros.giu@gmail.com

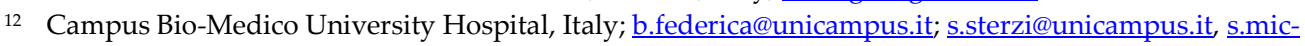
cinilli@unicampus.it, m.bravi@unicampus.it

13 National Center for Innovative Technologies in Public Health, Italian National Institute of Health, Rome, Italy 13; daniele.giansanti@iss.it, mauro.grigioni@iss.it

14 Padre Pio Foundation and Rehabilitation Centers, San Giovanni Rotondo, Italy; emanuele.f88@gmail.com, serena.diba@gmail.com

15 A.O.U. Città della Salute e della Scienza di Torino; monicasicari80@gmail.com, salvatore.petrozzino@gmail.com

16 CRRF “Mons. Luigi Novarese” Moncrivello, VC, Italy; csolaro@libero.it

17 Fondazione Don Carlo Gnocchi, Torino, Italy; stgargano@dongnocchi.it

18 Pontifical Gregorian University, Rome, Italy; benanti@unigre.it

19 Società Italiana di Medicina Fisica e Riabilitativa (SIMFER); paolobold@gmail.com, dbonaiuti2@yahoo.it

20 Paediatric Neurorehabilitation, Bambino Gesù Children's Hospital, Rome, Italy 20, Milan, Italy ; enrico.castelli@opbg.net

21 Department of Occupational and Environmental Medicine, Epidemiology and Hygiene, INAIL, Monte Porzio Catone, 00185 Rome, Italy; f.draicchio@inail.it

22 President Italian Federation of Persons with Spinal Cord Injuries (Faip Onlus), Rome, Italy; falabella@fishonlus.it

23 IRCCS Fondazione Don Carlo Gnocchi, Milan, Italy; sgaleri@dongnocchi.it, c.arienti@dongnocchi.it. 
24 Multidisciplinary Department of Medicine for Surgery and Orthodontics, University of Campania "Luigi Vanvitelli", Naples, Italy; francescagimigliano@gmail.com

25 Department of Electrical and Information Engineering, Politecnico di Bari, Italy; stefa no.mazzoleni@poliba.it

26 AULSS6 (Unique Sanitary Local Company) Euganea Padova, Rehabilitation Department, Italy; stefano.mazzon@gmail.com

27 Villa Beretta, Costa Masnaga, Italy; fmolteni@valduce.it

28 "Bambino Gesù" Children's Hospital - IRCCS, Movement Analysis and Robotics Laboratory MARlab, Rome, Italy; maurizio.petrarca@opbg.it

${ }^{29}$ Department of Neurosciences, Biomedicine and Movement Sciences, University of Verona, Verona, Italy 6; marialuisa.gandolfi@univr.it, alessandro.picelli@univr.it

30 Versilia Hospital AUSL Toscana Nord Ovest, Italy; federico.posteraro@uslnordovest.toscana.it

31 Presidente AITO (Associazione Italiana Terapisti Occupazionali);

32 Management Institute, Sant'Anna School of Advanced Studies, Pisa, Italy; giuseppe.turchetti@santannapisa.it

${ }^{33}$ Ferrara University Hospital, Neuroscience and Rehabilitation Department, Ferrara, Italy; $\underline{\text { so- }}$ fia.straudi@gmail.com

${ }^{34}$ Department of Medical and Surgical Specialties and Dentistry, University of Campania "Luigi Vanvitelli", Naples, Italy;

${ }^{35}$ IRCCS Fondazione Don Carlo Gnocchi, Rome, Italy;

36 Università degli studi di Torino, Torino, Italy 34;

* Correspondence: g.morone@hsantalucia.it (G.M.); Tel.: +39 0651501005; alessandro.desire@unicz.it (A.d.S.), tel: +390961712819 .

\begin{abstract}
The upper extremities limitation represents one of the essential functional impairments in patients with cervical spinal cord injury. Electromechanics assisted devices and robots are increasingly used in neurorehabilitation to help functional improvement in patients with neurological diseases. This review aimed to systematically report the evidence-based, state-of-art on clinical applications and robotic-assisted arm training (RAT) in motor and functional recovery in subjects affected by cervical spinal cord injury. The present study has been carried out within the framework of the Italian Consensus Conference on "Rehabilitation assisted by robotic and electromechanical devices for persons with disability of neurological origin" (CICERONE). PubMed/MEDLINE, Cochrane Library, and Physiotherapy Evidence Database (PEDro) databases were systematically searched from inception to September 2021. The 10-item PEDro scale assessed the study quality for the RCT and the AMSTAR-2 for the systematic review. Two different authors rated the studies included in this review. If consensus was not achieved after discussion, a third reviewer was interrogated. The 5-item Oxford CEBM scale was used to rate the level of evidence. A total of 11 studies were included. The selected studies were: two systematic reviews, two RCTs, one parallel-group controlled trial, one longitudinal intervention study and five case series. One RCT was scored as a high-quality study, while the systematic review was of low quality. RAT was reported as feasible and safe. Initial positive effects of RAT were found for arm function and quality of movement in addition to conventional therapy. The high clinical heterogeneity of treatment programs and the variety of robot devices could severely affect the generalizability of the study results; therefore, future studies are warranted to standardize the type of intervention and evaluate the role of roboticassisted training in subjects affected by cervical spinal cord injury.
\end{abstract}

Keywords: Cervical spinal cord injury; Arm function; Exoskeleton; Robot-assisted therapy; Robotic therapy; Rehabilitation.

\title{
1. Introduction
}

Spinal cord injury (SCI) represents one of the most disabling neurological conditions by complete or incomplete damage to the spinal cord with resulting detrimental consequences in motor, sensitive, and visceral controls. [1-3] 
The prevalence of SCIs widely varies among countries, ranging from 13.0 per million to 163.4 per million people. $[4,5]$ Considering that most of the presentation involves young adults, both sanitary costs and lifetime assistance costs are highly burdensome, estimating a comprehensive cost of more than 1 million dollars per person. [6] SCIs might arise from mechanical damages (i.e. contusions, compressions or lacerations of the spinal cord) or non-traumatic events (e.g. degenerative cervical myelopathies, cancers, infections, intervertebral disc diseases, etc.). [6,7]

High-level spinal cord lesions could lead subjects to a high disability, considering the loss of arms and hands function related to detrimental consequences of functional impairment, reduced independence in activities of daily living (ADL), and a poor HealthRelated Quality of Life (HRQoL). [1,2,7,8]

Rehabilitation might play a crucial role in the arm and hand functional recovery of patients affected by SCI, with a large variety of therapeutic options currently adopted. [7,9] It has been recently proposed that repetitive, task-specific, functional training could be considered effective in improving upper limb functions, even potentially interacting with the self-repair capacity of the spinal cord. [10,11]

Among the new therapeutic options, robotic devices are well suited to produce intensive, task-oriented motor training that might enhance conventional rehabilitation facilitating the plasticity-related recovery by increasing sensory feedback and supporting the motor system. [12]

These devices might perform arm or hand-assisted training, typically targeting either the shoulder and elbow, or the wrist and fingers. Robotic devices can be categorized as exoskeletons or end-effectors. Exoskeletons are devices that directly control the articulation of targeted joint(s), whereas robotic end-effectors contact users at the distal part of their limb. $[11,13,14]$ Robotic devices are currently used in clinical practice to deliver an adequate intensity of training in terms of movement repetitions even in more severe subjects, which promotes functional recovery and may potentially facilitate adaptive plasticity. [11,13]

In addition, robotic training provides the standardized rehabilitative training and monitors recovery of motor function in patients more objectively, thus reducing the subjective human influence. [15] Robotic rehabilitation aims to optimize learning strategies and to provide a patient-tailored rehabilitation plan. [11] Nowadays, more than 120 devices have been developed for upper limb rehabilitation of patients affected by neurologic disability. [16]

To date, an increased interest has been growing in the scientific literature, with several papers suggesting medical relevant features of robotic-assisted rehabilitation in functional recovery of patients affected by neurologic disability. [14,17-19] However, despite these promising findings, there is not agreement on the effectiveness of this novel approach in the current clinical practice of the rehabilitation field. Moreover, even the expensive technology could limit the spreading of this advanced treatment in clinical settings and the evidence of its effectiveness in patients affected by neurological diseases of rehabilitative interest, including SCI.

Therefore, this comprehensive review of systematic reviews and clinical studies summarizes the state-of-art on safety, clinical applications, and effectiveness of robotic rehabilitation in the integrated management of upper limb functional recovery in SCI patients. 


\section{Materials and Methods}

The present study has been carried out within the framework of the Italian Consensus Conference on "Rehabilitation assisted by robotic and electromechanical devices for persons with disability of neurological origin" (CICERONE). [20]

Search strategy

PubMed/MEDLINE, Cochrane Library, and Physiotherapy Evidence Database (PEDro) databases were systematically searched from inception to September, 2021 for all the papers published following the SPIDER tool strategy, [21] depicted by Table I.

This comprehensive systematic review of systematic reviews and clinical studies has been performed in accordance with the Preferred Reporting Items for Systematic Reviews and Meta-analyses (PRISMA) statement. [22]

\section{Selection criteria}

After the 'duplicates' removal, two reviewers (LP, LL) independently screened for inclusion title and abstract of all potentially relevant studies identified. In case of disagreement, a consensus was achieved by the decision of a third reviewer (AdS). Full-text studies were retrieved by the same two reviewers (LP, LL) and independently screened for inclusion. If consensus was not achieved by discussion between them, disagreements were solved by the decision of a third reviewer (AdS).

Randomized controlled trials were considered eligible if responding to the questions defined according to the following PICO model: P) Participants: SCI patients in acute, subacute ( $\leq 3$ months after injury), or chronic phase; I) Intervention: Rehabilitation training with robotic-assisted devices for upper limb, with or without conventional therapy; C) Comparator: Conventional rehabilitation; O) Outcome measures: safety of robotic rehabilitation, the feasibility of robotic rehabilitation, upper limb strength, functioning, independence in ADL, and HRQoL.

We included systematic reviews, randomized controlled trials (RCTs), observational analytic studies, and case series. Exclusion criteria were: 1) papers involving animals; 2 ) language other than English; 3) case reports design; 4) participants with different neurologic disabilities from SCI; 5) robotic-assisted rehabilitation combined with other advanced technologies such as non-invasive brain stimulations (NIBS) or transcranial direct current stimulation ( $\mathrm{tDCS}$ ).

\section{Data extraction and synthesis}

All data were extracted from eligible full-text documents through Excel by two different authors. In case of disagreement, the consensus was achieved by the review of a third author.

The following data were extracted: 1) title; 2) authors; 3) publication year; 4) study design; 5) participants; 6) intervention characteristics; 7) outcomes; 8) main findings.

All studies included were synthesized, describing both study characteristics and data extracted. A meta-analysis was not performed given the high clinical heterogeneity in design, intervention, and outcomes assessed in the different studies. 


\section{Study quality}

The 5-item Oxford CEBM scale was used to rate the level of evidence (OCEBM website). The study quality included was assessed by the 16-item assessment of multiple systematic reviews 2 (AMSTAR 2) scale [23] for systematic reviews, and the 10-item PEDro scale24 for the randomised clinical trials. Regarding the PEDro scale, the risk of bias was rated as poor (0-3), fair (4-5), good (6-8) and excellent (9-10) in line with the PEDro scale. [24] Two different authors rated the studies included in this systematic review. If consensus was not achieved after discussion, a third reviewer was interrogated.

\section{Results}

\section{Evidence synthesis}

Out of 226 studies identified from the databases, 214 were considered eligible for inclusion after duplicate removal and screened for title and abstract: 164 were excluded, and 50 full-text papers were screened. Subsequently, 39 articles were excluded because they did not respect eligibility criteria. As a result, 11 papers were included in the qualitative synthesis (PRISMA flow diagram was depicted by Figure 1): 5 case series, [25-29] 1 parallel-group controlled trial, [30] 2 RCT, [31,34] 2 systematic reviews [32,33] and one longitudinal intervention study. [35]

The studies included in this systematic review were published from 201225 to 202034, covering several Nations from all over the world; more in detail, seven studies were from the Americas (two from Canada [25,32] and five from USA [26,27,29,30,33]), two from Europe (1 from Netherlands [28] and one from UK [35]), and two from Asia (Republic of Korea [31,34]).

Evidence level and study quality of the included studies

Due to the high clinical heterogeneity of the included studies; thus, the results are described qualitatively. Based on the Oxford Centre for Evidence-Based (OCEBM) 2011 Levels of Evidence, [34] we included two systematic reviews [32,33] (Level 1), 2 RCT [31,34] (Level 2), one parallel-group controlled trial [30] (Level 3), one longitudinal intervention trial [35] (Level 3), and five case series (Level 4). [25-29]

The study cohort sample sizes were highly heterogeneous in the research studies, ranging from 5 (case series) [28] to 34 (RCT) [31] for clinical trials; nevertheless, the systematic reviews included larger samples (73 study participants by Singh et al. [32] and 88 by Yozbatiran et al. [33]). All the studies assessed patients of both genders, with ages ranging from 17 [26] to 76 years. [29] The study by Fitle et al. [27] did not report age.

Concerning the study quality of the clinical studies, we reported one good-quality [31], one fair-quality [34], according to the PEDro scale. [24] The two systematic reviews showed a low quality [31] and a critically low quality [33] according to AMSTAR 2 scale.[23]

Clinical characteristics of study participants

Six studies included SCI patients in the chronic phase [26,27-30,34,] two in the subacute phase [25,35], and three papers [31-33] included both chronic and subacute SCI patients. Complete (American Spinal Injury Association Impairment Scale - AIS - A and B) and incomplete lesions (AIS C and D) were assessed by seven studies [25,26,28,31-34] while four studies $[27,29,30,35]$ selected only incomplete lesions (AIS C and D). All the clinical trials included clarified SCI levels, ranging between C2 [29] and C8 [31] (further details are depicted in Table II). 
Robotic rehabilitation characteristics

Robotic devices assessed in the studies included resulted to be extremely heterogeneous. Armeo Spring, [25,32,33] InMotion 3.0 Wrist robot, [26,32] Haptic Master, [28,32] MAHI Exo-II, [27,29,30,33] Armeo Power, [31,34] RiceWrist-S, [33] Reo Go, [32,33] Haptic Master, [32,33] Reaching Robot, [32,33] Amodeo, [34] SEM Glove. [35]

The joints involved by robotic training were: shoulder, [25,34] elbow, [25,27,29-31,34] wrist, [25,27,29-31,34] and fingers. [31,34-35] Even robotic-assisted rehabilitation programs were heterogeneous, varying from 429, [31] to 12 weeks [35], with the duration of the interventions ranging from a total of 30 minutes [31] to 4 hours per day. [35] Training sessions ranged from 130 to 5 per week. [25] On the other hand, supervision was not clarified by two study [26,34], one did not perform a supervision35, whereas all the other research studies included assessed supervised exercise programs. [25,27-31] Robot-assisted training was assessed as an add-on conventional therapy in four studies, $[25,28,31,34]$, whereas five studies considered stand-alone robotic training. $[26,27,29,30,35]$ Only two studies compared occupational therapy with occupational therapy combined with robotic training. [31,34] Moreover, Zariffa et al. [25] compared the efficacy of the unilateral treatment with the contralateral upper limb. Lastly, both systematic reviews included studies with robotic training combined or not combined with conventional therapy (further details on robotic rehabilitation in the included studies are depicted by Table III). [32,33]

Main findings of the included studies

All the case series [25-29] included in the present systematic review assessed the feasibility of robotic rehabilitation in SCI patients. Zariffa et al. [25] assessed both compliance and therapist timing, reporting that more rehabilitation exercises were performed with progressively less hands-on involvement by the therapist. Tolerance has been assessed by Francisco et al. [29], reporting no significant increase of self-reported pain and discomfort level during the therapy sessions. Accordingly, Cortes et al. [26] reported a high safety profile and tolerance without increasing pain and spasticity, and Vanmulken et al. [28] showed a discrete tolerance (Usefulness, Satisfaction and Ease-of-use questionnaire mean score of $66.1 \pm 14.7 \%$ ). Lastly, it should be highlighted that all papers included in this systematic review [25-33] did not report any major adverse event during robot-assisted training in SCI patients.

A main rehabilitative measure as muscle strength was assessed by all the research articles, albeit with a wide heterogeneity in terms of outcomes, including Medical Research Council grade, [29] Manual Muscle Test (MMT), [31] grip [25,29] and pinch [29] strength, and upper extremity motor score (UEMS) [25]. Two studies [25,26] reported no significant changes in terms of muscle strength; on the contrary, Francisco et al. [29] showed a significant improvement of muscle strength (UEMS: $31.5 \pm 2.3$ vs $34.0 \pm 2.3 ; \mathrm{p}=0.04$; grip strength: $9.7 \pm 3.8$ vs $12 \pm 4.3$; $\mathrm{p}=0.02$; pinch strength $4.5 \pm 1.1$ vs $5.7 \pm 1.2 ; \mathrm{p}=0.01$ ), even maintained at follow-up evaluation (UEMS: $35.5 \pm 2.0 ; \mathrm{p}=0.02$; grip strength: $12.7 \pm 4.0 ; \mathrm{p}=0.05$; pinch strength $5.6 \pm 1.2 ; \mathrm{p}=0.02)$.

Kim et al., 31 the only RCT included in this systematic review, reported a significant improvement in terms of UEMS in the robotic training group compared to the control group ( 1 [0 to 3$]$ vs 0 [ -1 to 1$] ; p=0.03$ ). However, no significant changes in MRC scale were shown. In particular Elbow flexors (C5) changed from 0 [0 to 1] to 0 [ -0.82 to 0 ] $(p=0.21)$, wrist extensors $(C 6)$ changed from 0 [0 to 1$]$ to $0[-0.5$ to 0$](p=0.08)$, elbow extensors (C7) changed from 0 [0 to 1$]$ to 0 [0 to 0$](p=0.16)$, finger flexors changed from 0 [0 to 1$]$ to 0 [0 to 0.5$](\mathrm{p}=0.66)$, and 5 th finger abductors (T1) changed from 0 [0 to 1$]$ to 0 [0 to 0.08 ] $(\mathrm{p}=0.59)$. 
In line with previous findings, both systematic reviews [32,33] affirmed that evidence supporting robot-assisted training effectiveness in muscle strength improvement in SCI patients is still controversial.

Concerning functioning, several outcome measures were assessed by the included papers, including Graded and redefined assessment of strength, sensibility, and prehension (GRASSP) [25,30], Action Research Arm Test (ARAT), [25,27,29,30] Jebsen-Taylor Hand Function Test (JTHFT), [27,29] SCIM II, [29] and SCIM III [31]. Zariffa et al. [25] showed a significant improvement of GRASSP score only in the subgroup with partial hand function at baseline ( $6.0 \pm 1.6$ vs $1.9 \pm 0.9 ; p=0.04)$. Considering the whole sample, no significant results $(p>0.05)$ were underlined in both GRASSP scores. On the contrary, Frullo et al. [30] reported significant results in GRASSP strength $(\mathrm{p}=0.031)$ and GRASSP sensation (0.002), although these results have not been corrected for multiple comparisons. No significant effects were shown in the ARAT score $(\mathrm{p}=0.128)$. However, Francisco et al. [29], in their case series, reported a significant increase in terms of ARAT (30.7 \pm 3.8 vs $34.3 \pm 4.0 ; p=0.02)$ and JTHFT $(0.14 \pm 0.04$ vs $0.21 \pm 0.07 ; p=0.04)$, whereas SCIM II did not significantly improve (62.1 \pm 9.7 vs $62.6 \pm 9.7 ; \mathrm{p}=0.18)$.

On the other hand, the RCT performed by Kim et al. reported significant differences between groups in terms of total SCIM-III score (7 [2 to 11] vs 0 [ -4 to 4 ]; $p<0.01$ ), however only the mobility (room and toilet) item significantly varied between groups (1 [0 to 3] vs 0 [-1 to 1$] ; \mathrm{p}=0.02)$, in contrast with the other items not showing significant differences.31

Both systematic reviews $[32,33]$ reported that robot-assisted rehabilitation might be considered promising training to improve muscle function in SCI.

Lastly, the case series performed by Cortes et al. [26] evaluated kinematics and corticospinal excitability after robotic rehabilitation in SCI patients. The authors reported a significant improvement of kinematic ( $1.17 \pm 0.11$ radians vs $1.03 \pm 0.08$ radians; $\mathrm{p}=0.03)$ and smoothness of movement $(0.26 \pm 0.03$ vs $0.31 \pm 0.02 ; \mathrm{p}=0.03)$ in SCI patients; however, the corticospinal excitability did not show significant changes (amplitude: $32 \pm 0.5 \mathrm{mV}$ vs $27 \pm 0.06 \mathrm{mV}$; $\mathrm{p}=0.35$; latency: $17.4 \pm 0.7 \mathrm{~ms}$ vs $16.9 \pm 0.74 \mathrm{~ms} ; \mathrm{p}=0.28$ ). Similarly, Fitle et al. [27] showed a significant improvement between pre- and post-intervention in the nonsegmental kinematic measure (normalized speed) of the less affected arm ( $p=0.01)$. In addition, segmental kinematic measures improved significantly in the more affected arm $(\mathrm{p}=0.03)$.

Lastly, the study by Frullo et al. [30] reported a significant improvement of normalized speed $(\mathrm{p}<0.001)$, mean arrest period ratio $(\mathrm{p}=0.001)$, and spectral arc length $(\mathrm{p}=0.001)$ only in the assist-as-need group. 




Figure 1. Prisma Flow chart 
Table I. Spider tool search strategy.

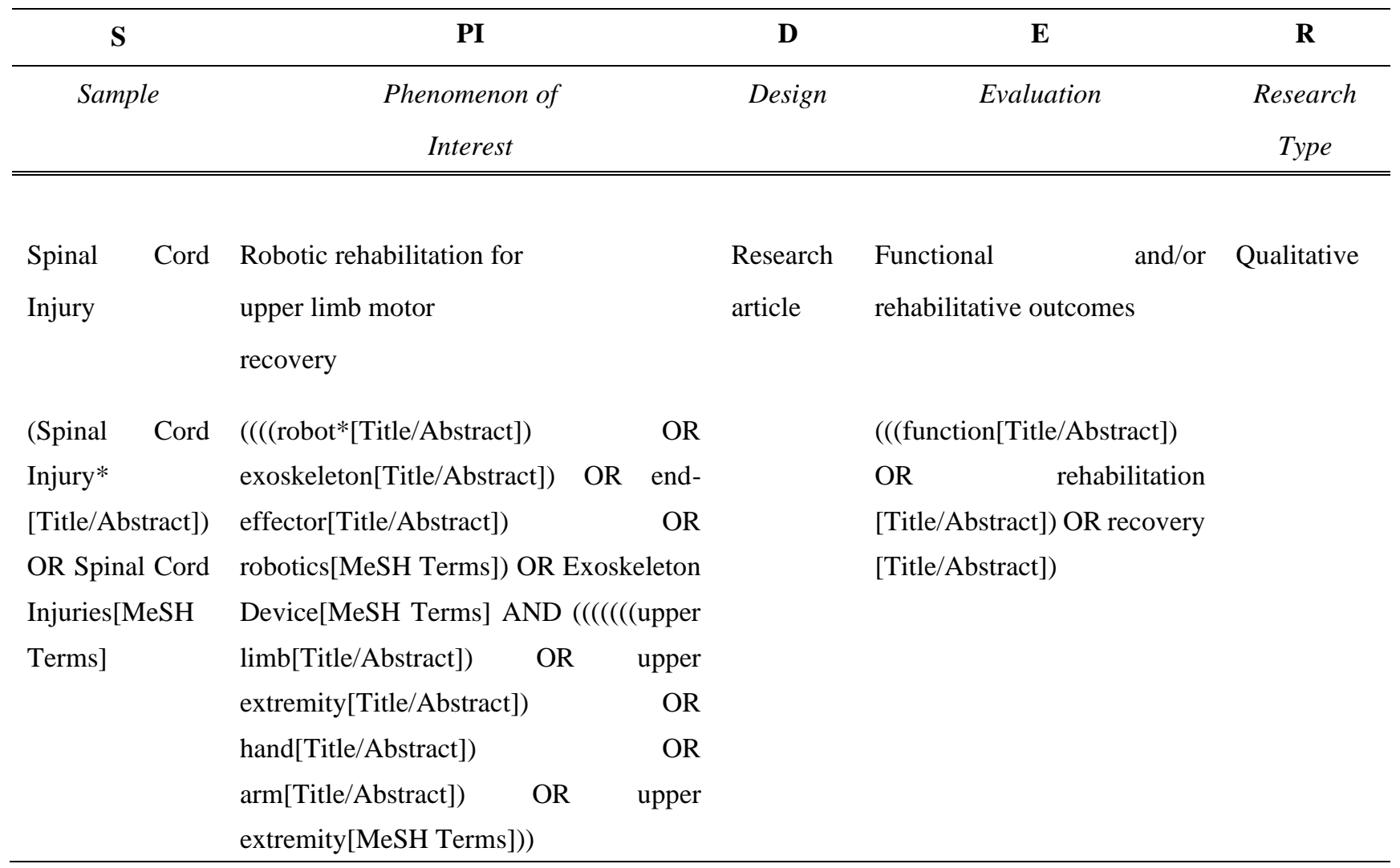


Table II. Main characteristics of the studies included in this systematic review.

\begin{tabular}{|c|c|c|c|c|c|c|c|c|c|c|}
\hline Article & Nation & Design & Aim & $\begin{array}{c}\text { Numberparticip } \\
\text { ants } \\
\text { (drop-outs) }\end{array}$ & Gender and age & SCI Stage & SCI according to AIS & $\begin{array}{l}S C I \\
\text { level }\end{array}$ & $\begin{array}{c}\text { Methodologica } \\
\text { l quality }\end{array}$ & CBIM \\
\hline Zariffa $2012^{25}$ & Canada & Case Series & $\begin{array}{l}\text { To assess the feasibility and } \\
\text { efficacy of upper limb robotic } \\
\text { rehabilitation device in subacute } \\
\text { cervical SCI }\end{array}$ & $15(3)$ & $\begin{array}{c}14 \mathrm{M}, 1 \mathrm{~F} \\
19-75 \text { years }\end{array}$ & Subacute & $\begin{array}{l}\text { AIS A }(n=2) \\
\text { AIS B }(n=4) \\
\text { AIS C }(n=1) \\
\text { AIS D }(n=5)\end{array}$ & C4-C6 & $\mathrm{n} / \mathrm{a}$ & 4 \\
\hline Cortes $2013^{26}$ & USA & Case Series & $\begin{array}{l}\text { To assess feasibility, safety, and } \\
\text { effectiveness of robotic-assisted } \\
\text { training in chronic SCI }\end{array}$ & $10(0)$ & $\begin{array}{c}8 \mathrm{M}, 2 \mathrm{~F} \\
17-70 \text { years }\end{array}$ & Chronic & $\begin{array}{l}\text { AIS A }(n=3) \\
\text { AIS B }(n=4) \\
\text { AIS C }(n=1) \\
\text { AIS D }(n=2)\end{array}$ & $\mathrm{C} 4-\mathrm{C} 6$ & $\mathrm{n} / \mathrm{a}$ & 4 \\
\hline Fitle $2015^{27}$ & USA & Case series & $\begin{array}{l}\text { To assess feasibility and } \\
\text { effectiveness of a robotic } \\
\text { exoskeleton designed to train } \\
\text { elbow, forearm and wrist } \\
\text { movements }\end{array}$ & $10(2)$ & $\begin{array}{l}8 \mathrm{M}, 2 \mathrm{~F} \\
\text { age: NR }\end{array}$ & Chronic & AIS C-D (n=2) & $\mathrm{C} 2-\mathrm{C} 6$ & $\mathrm{n} / \mathrm{a}$ & 4 \\
\hline
\end{tabular}




\section{Vanmulken $2015^{28}$}

Netherlands

Case Series

effectiveness (arm-hand function

and performance) of haptic robot

technology

To assess feasibility, tolerability,

$$
\text { and }
$$

Francisco $2017^{29}$

USA

Case Series

effectiveness of robotic-assisted

$$
\text { arm training }
$$

$$
\begin{gathered}
\text { 8 M, } 2 \text { F, } \\
\text { 19-76 years }
\end{gathered}
$$

Chronic

AIS C ( $(n=4)$

AIS D (n=4)

C2-C7

\section{Parallel}

group

controlled

trial

To assess feasibility of subject-

adaptive robotic-assisted therapy:

AAN vs ST training modality

17 (3)

\section{$12 \mathrm{M}$ and $2 \mathrm{~F}, 3$}

$\mathrm{NR}$
53.5 years

$28 \mathrm{M}, 6 \mathrm{~F}$,

To assess the clinical efficacy of

Republic of

Korea

RCT

upper limb robotic therapy in

people with tetraplegia

RT: 17 (2)

CT: 17 (2)

RT: $56.7 \pm 13.6$

years

CT: $47.1 \pm 14.9$

years

To summarize feasibility and

Singh $2018^{32}$

Canada

Systematic

review

outcomes of robotic-assisted

upper extremity training for

patients with cervical SCI

To summarize the current

Yozbatiran $2019^{33}$

USA

Systematic

review

evidence of robot-assisted

rehabilitation in patients with

$$
\text { tetraplegia }
$$

To assess the effects of combined

Jung $2019^{34}$
RCT
Republic of

Korea upper limb robotic therapy (RT)

as compared to conventional
73 (11)

$$
\begin{aligned}
& 46 \mathrm{M}, 8 \mathrm{~F}, 7 \mathrm{NR} \text { Subacut } \\
& 17-75 \text { years }
\end{aligned}
$$

c

AIS A-B $(n=16)$

AIS C-D $(n=46)$

C2-C8

Critically low

quality

AIS A-B(n=14)

69 M, 13 F, 6 NR Subacute/Chroni 17-76 years

c

AIS C-D $(\mathrm{n}=58)$

$3 \mathrm{NR}$

AIS A (n=3)

AIS B ( $n=4)$

AIS C ( $n=7)$
RT: 22 (5) RT: $47.23 \pm 14 \quad$ Subacute

CT: 16 (3) 
occupational therapy (OT) in SCI

$$
\text { patients }
$$

Intervention

To investigate the therapeutic

al

Osuagwu $2020^{35}$

UK longitudinal effect of a self-administered

home-based hand rehabilitation

clinical trial

design programme for people with

cervical SCI using the soft extra
AIS D ( $n=16)$

$15(0)$

$11 \mathrm{M}, 4 \mathrm{~F}$

$50.3(33-60)$

Chronic

AIS C ( $n=3)$

AIS D ( $\mathrm{n}=11)$

C2-C5

$\mathrm{n} / \mathrm{a}$ muscle (SEM) Glove

Abbreviations: $A A N$ : assist-as-needed; $A I S$ : American Spinal Injury Association Impairment Scale; $C T$ : conventional therapy; F: Female; $M$ : Male; NR: not reported; RCT: Randomized Controlled Trial; $R T$ : robotic

training; SCI: Spinal Cord Injury; ST: subject-triggered; USA: United States of America; CBIM 
Table II. Main characteristics of the studies included in this systematic review.

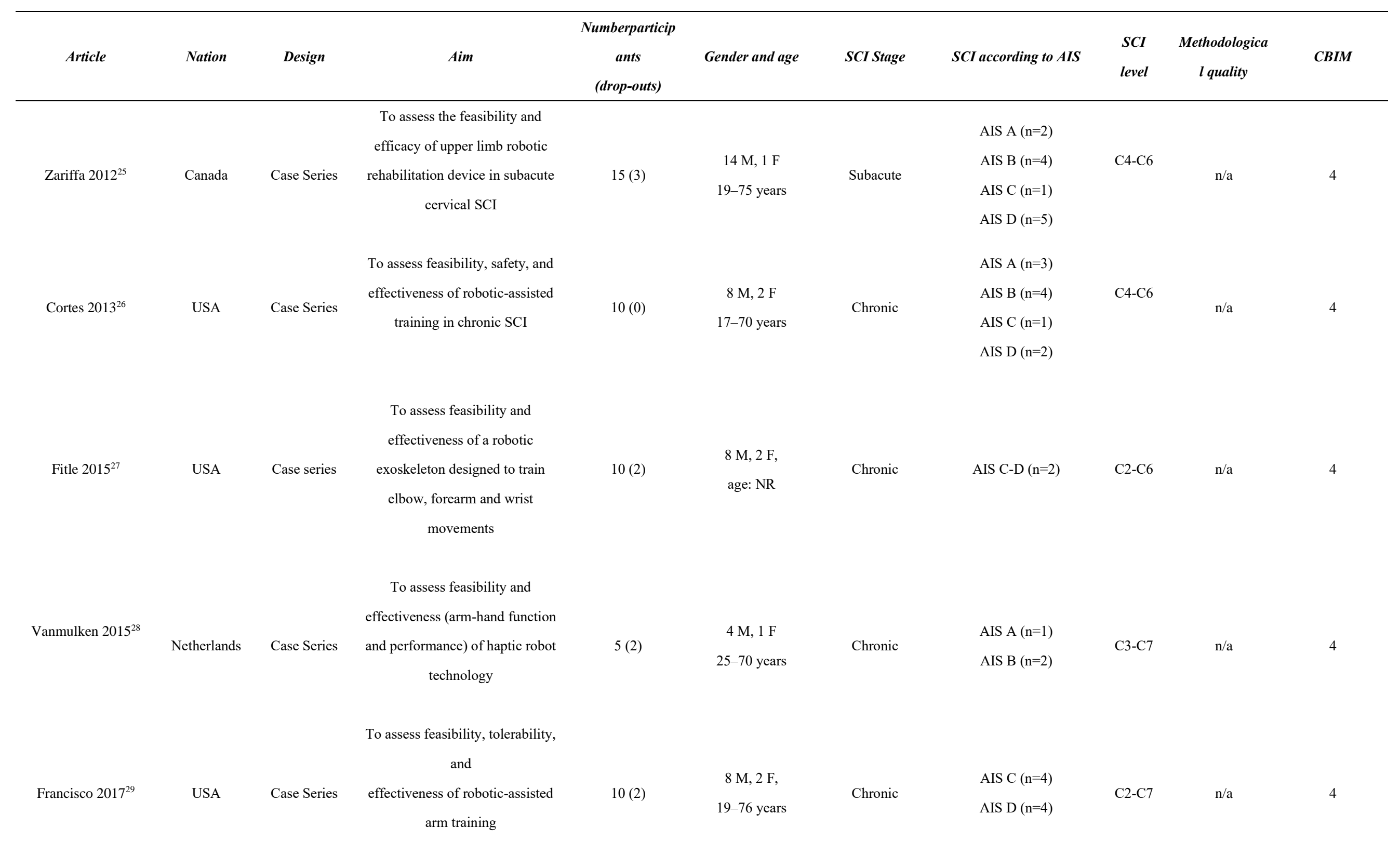




\begin{tabular}{|c|c|c|c|c|c|c|c|c|c|c|}
\hline Frullo $2017^{30}$ & USA & $\begin{array}{c}\text { Parallel } \\
\text { group } \\
\text { controlled } \\
\text { trial }\end{array}$ & $\begin{array}{l}\text { To assess feasibility of subject- } \\
\text { adaptive robotic-assisted therapy: } \\
\text { AAN vs ST training modality }\end{array}$ & $17(3)$ & $\begin{array}{c}12 \mathrm{M} \text { and } 2 \mathrm{~F}, 3 \\
\mathrm{NR} \\
53.5 \text { years }\end{array}$ & Chronic & AIS C $-\mathrm{D}(\mathrm{n}=17)$ & $\mathrm{C} 3-\mathrm{C} 8$ & $\mathrm{n} / \mathrm{a}$ & 4 \\
\hline $\operatorname{Kim} 2019^{31}$ & $\begin{array}{c}\text { Republic of } \\
\text { Korea }\end{array}$ & RCT & $\begin{array}{l}\text { To assess the clinical efficacy of } \\
\text { upper limb robotic therapy in } \\
\text { people with tetraplegia }\end{array}$ & $\begin{array}{c}34(4) \\
\text { RT: } 17 \text { (2) } \\
\text { CT: } 17 \text { (2) }\end{array}$ & $\begin{array}{c}28 \mathrm{M}, 6 \mathrm{~F}, \\
\text { RT: } 56.7 \pm 13.6 \\
\text { years } \\
\text { CT: } 47.1 \pm 14.9 \\
\text { years }\end{array}$ & $\begin{array}{c}\text { Subacute/Chroni } \\
\text { c }\end{array}$ & $\begin{array}{l}\text { AIS A }(n=8) \\
\text { AIS B }(n=6) \\
\text { AIS C }(n=4) \\
\text { AIS D }(n=16)\end{array}$ & $\mathrm{C} 2-\mathrm{C} 8$ & $8 / 10$ & 2 \\
\hline Singh $2018^{32}$ & Canada & $\begin{array}{c}\text { Systematic } \\
\text { review }\end{array}$ & $\begin{array}{l}\text { To summarize feasibility and } \\
\text { outcomes of robotic-assisted } \\
\text { upper extremity training for } \\
\text { patients with cervical SCI }\end{array}$ & $73(11)$ & $\begin{array}{c}46 \mathrm{M}, 8 \mathrm{~F}, 7 \mathrm{NR} \\
17-75 \text { years }\end{array}$ & $\begin{array}{c}\text { Subacute/Chroni } \\
\text { c }\end{array}$ & $\begin{array}{l}\text { AIS A-B }(n=16) \\
\text { AIS C-D }(n=46)\end{array}$ & $\mathrm{C} 2-\mathrm{C} 8$ & $\begin{array}{c}\text { Critically low } \\
\text { quality }\end{array}$ & 3 \\
\hline Yozbatiran $2019^{33}$ & USA & $\begin{array}{c}\text { Systematic } \\
\text { review }\end{array}$ & $\begin{array}{l}\text { To summarize the current } \\
\text { evidence of robot-assisted } \\
\text { rehabilitation in patients with } \\
\text { tetraplegia }\end{array}$ & $88(13)$ & $\begin{array}{c}69 \mathrm{M}, 13 \mathrm{~F}, 6 \mathrm{NR} \\
17-76 \text { years }\end{array}$ & $\begin{array}{c}\text { Subacute/Chroni } \\
\text { c }\end{array}$ & $\begin{array}{l}\text { AIS A-B(n=14) } \\
\text { AIS C-D }(n=58) \\
3 \mathrm{NR}\end{array}$ & $\mathrm{C} 2-\mathrm{C} 7$ & Low quality & 3 \\
\hline Jung $2019^{34}$ & $\begin{array}{c}\text { Republic of } \\
\text { Korea }\end{array}$ & RCT & $\begin{array}{l}\text { To assess the effects of combined } \\
\text { upper limb robotic therapy (RT) } \\
\text { as compared to conventional } \\
\text { occupational therapy (OT) in SCI } \\
\text { patients }\end{array}$ & $\begin{array}{c}38(8) \\
\text { RT: } 22(5) \\
\text { CT: } 16(3)\end{array}$ & $\begin{array}{c}24 \mathrm{M}, 6 \mathrm{~F} \\
\text { RT: } 47.23 \pm 14 \\
\text { CT: } 53 \pm 13.5\end{array}$ & Subacute & $\begin{array}{l}\text { AIS A }(n=3) \\
\text { AIS B }(n=4) \\
\text { AIS C }(n=7) \\
\text { AIS D }(n=16)\end{array}$ & $\mathrm{C} 2-\mathrm{C} 7$ & $4 / 10$ & 3 \\
\hline Osuagwu $2020^{35}$ & UK & $\begin{array}{l}\text { Intervention } \\
\qquad \text { al } \\
\text { longitudinal } \\
\text { clinical trial } \\
\text { design }\end{array}$ & $\begin{array}{l}\text { To investigate the therapeutic } \\
\text { effect of a self-administered } \\
\text { home-based hand rehabilitation } \\
\text { programme for people with } \\
\text { cervical SCI using the soft extra } \\
\text { muscle (SEM) Glove }\end{array}$ & $15(0)$ & $\begin{array}{c}11 \mathrm{M}, 4 \mathrm{~F} \\
50.3(33-60)\end{array}$ & Chronic & $\begin{array}{l}\text { AIS C (n=3) } \\
\text { AIS D (n=11) } \\
\text { Untested (n=1) }\end{array}$ & $\mathrm{C} 2-\mathrm{C} 5$ & $\mathrm{n} / \mathrm{a}$ & 4 \\
\hline
\end{tabular}

Abbreviations: $A A N$ : assist-as-needed; $A I S$ : American Spinal Injury Association Impairment Scale; $C T$ : conventional therapy; F: Female; $M$ : Male; NR: not reported; $R C T$ : Randomized Controlled Trial; $R T$ : robotic

training; SCI: Spinal Cord Injury; ST: subject-triggered; USA: United States of America; CBIM 


\section{Discussion}

Advancement in technology has been widely spreading in the rehabilitation field during the past two decades, and SCI patients might benefit from robotic rehabilitation. However, albeit this approach is commonly adopted in the clinical practice, this systematic review showed that only a few studies assessed the effectiveness of robotic-assisted training for recovering upper limb muscle strength and function in patients with SCI.

Taken together, our findings suggested that robotic devices for upper limbs might be considered safe, tolerable, and feasible in the complex rehabilitative management of SCI patients. However, to date, safety, tolerance, and feasibility of robot-assisted training have been primarily investigated in patients with other neurological diseases (i.e. stroke and multiple sclerosis) $[37,38]$ and these outcomes should be deeply assessed in SCI patients, starting from the findings reported by the present systematic review.

We highlighted that robotic rehabilitation mainly was assessed in patients suffering from incomplete SCI, both with sub-acute [25,31-34] and chronic lesions. [26-33,35] Among the included studies, Zariffa et al. [25] suggested that SCI patients with more represented residual function could beneficiate from robot-assisted therapy more than other subjects.

Indeed, considering that plasticity-related recovery could be enhanced by intensive and task-oriented motor training, [11-14] motor and sensory feedback stimuli might be more effective in patients with incomplete SCI than complete SCI, suggesting a potential positive effect robotic-assisted training. However, in literature, the role of different therapies has been assessed, considering that sensory and motor afferent stimuli were not the only ones involved in the plasticity process. More in detail, Yozbatiran et al. [39] suggested that modulating excitatory input of the corticospinal tracts on spinal circuits induced by tDCS combined with robot-assisted training could improve arm and hand functions in persons with incomplete SCI. This intriguing study has not been included in our systematic review, considering that the combination of robotic-assisted rehabilitation with other advanced technologies (i.e. NIBS and tDCS) was an exclusion criterion due to the limitation that they might affect the efficacy of robot-assisted training. However, we are aware that this combination should be deeply investigated in future studies on SCI patients.

Robotic training should be considered as an "add on" to conventional therapy in subacute SCI patients ( $\leq 3$ months after injury); four studies included in this systematic review assessed the role of robotic-assisted rehabilitation combined with conventional physical therapy $[25,28]$ and occupational therapy, $[25,31]$ probably due to complex scenario underpinning SCI management. In contrast, robotic treatment has been proposed as a stand-alone therapy in 3 case series out of 4 involving chronic SCI patients. $[26,27,29]$

The present comprehensive systematic review showed a lack of evidence on differences between proximal (shoulder elbow) and distal (hand) training according to the robot design. More in detail, rehabilitation robots could be classified into two groups: endeffector based robots, which provide training capability encapsulating a large portion of the functional workspace, and exoskeletons, designed to resemble human anatomy with a structure enabling individual actuation of joints. [40] Therefore, we would like to highlight that future studies should involve enhanced control modes to allow additional treatment options in SCI patients; indeed, taking into account the different actions that the upper limb might exert (i.e. reaching and grasping), robotic devices might have a more targeted function with a more specific mechanical design in order to perform an adequate patient-tailored rehabilitation in subjects after SCI. 
Concerning the type of intervention proposed, very high variability was recorded in terms of robot devices, the number of sessions per day, session duration, frequency, and joint involvement. This intrinsic limitation, probably related to the first phase of adopting new technology, severely affects the generalizability of these findings. In addition, it should be noted that the type of treatment intervention should be based on the SCI level, considering the clinical heterogeneity of functional disability occurring in cervical SCI. Future studies should focus on larger samples involving cervical SCI patients divided into subgroups to provide a patient-tailored robotic rehabilitative treatment.

In the literature, we found two similar systematic reviews investigating the role of robotic rehabilitation in SCI patients, albeit their quality was classified as low [32] and very low, [33] according to AMSTAR 2 scale. [23] Indeed, both Smith et al.32 and Yozbatiran et al. [33] summarized the available literature on the robot-assisted training in upper limb rehabilitation of SCI patients, including even case reports and studies on the combination of robotic rehabilitation with other advanced technologies, severely affecting the homogeneity of data assessed and heavily influencing their results.

Nevertheless, by the present systematic review, the RCT performed by Kim et al. [31] was investigated first. This good-quality paper reported a significant improvement in terms of UEMS in the robotic training group compared to the control group ( 1 [0 to 3] vs 0 [ -1 to 1$] ; \mathrm{p}=0.03$ ) in SCI patients; on the other hand, no significant changes in MRC scale were showed $(\mathrm{p}>0.05)$. The authors suggested that significant improvement in muscle strength might have potential benefits in terms of short-distance mobility and electrical wheelchair manipulation. In line with these findings, significant improvements in SCIM-III scores (7 [2 to 11] vs 0 [ -4 to 4 ]; $\mathrm{p}<0.01$ ) in the robot-assisted rehabilitation group might have positive effects in terms of independence in the ADL. [31]

Considering these findings, the present study might be viewed as the first systematic review performed by a large consensus panel of experts, including research studies specifically assessing the effects of robot-assisted training of the upper limb in patients with SCI. We showed that the current available literature on this topic might be defined as low-quality evidence. The lack of evidence might be partly due to the rapid evolution of advanced technologies with high costs that might not allow a standardization and reproducibility of single large-scale rehabilitation intervention.

The studies included in this systematic review had several limitations, as the small sample sizes, [25,26,31] the lack of a control group, [25-29] the monocentric design $[26,27,30,31]$ and the lack of long-term follow up evaluations; [25-27,28,31] as well as the wide variability in robotic devices, training protocols, and outcome measures adopted in the studies.

\section{Conclusions}

Taken together, the present comprehensive systematic review summarized the state-ofthe-art of robotic-assisted rehabilitation treatment in patients suffering from cervical SCI. Nowadays, robotic-assisted training is still experimental, but recent studies provided preliminary evidence showing intriguing positive effects on functional outcomes in SCI patients. We are aware that the high clinical heterogeneity of treatment programs and the variety of robot devices could severely affect the generalizability of the study results; therefore, future studies are warranted to standardize the type of intervention and evaluate the role of a robot-assisted training in the complex rehabilitation management of patients with SCI.

Author Contributions: GM, AdS, LP,SS conceptualized paper, performed and/or supervised research and drafted manuscript; AdS, LP, LL,SS performed quality assessment of the guidelines; CA, SS, as all organization committee and scientific technical committee of the CICERONE consensus conference supervises research, read and corrected manuscript. All authors read and approved the final version of the manuscript. 


\section{Conflicts of interest}

The authors certify that there is no conflict of interest with any financial organization regarding the material discussed in the manuscript.

\section{Funding}

The authors report no involvement in the research by the sponsor that could have influenced the outcome of this work.

Acknowledgments: The present study has been carried out within the framework of the Italian Consensus Conference on "Rehabilitation assisted by robotic and electromechanical devices for persons with disability of neurological origin" (CICERONE), promoted by the Italian Society of Physical and Rehabilitation Medicine (SIMFER, Società Italiana di Medicina Fisica e Riabilitativa) and Italian Society of Neurological Rehabilitation (SIRN, Società Italiana di Riabilitazione Neurologica).

Group name Upper limb working group within the "Italian Consensus Conference on rehabilitation assisted by robotic and electromechanical devices for persons with disability of neurological origin" (CICERONE): Michela AGOSTINI (Department of Neuroscience, section of rehabilitation, University-General Hospital of Padova, Italy); Irene APRILE (IRCCS Fondazione Don Carlo Gnocchi ONLUS Florence, Italy); Ludovica BALUARDO (Ferrara University Hospital, Neuroscience and Rehabilitation Department, Ferrara, Italy); Alberto BATTISTINI (IRCCS Istituto delle Scienze Neurologiche di Bologna, UOC Medicina Riabilitativa e Neuroriabilitazione, Bologna, Italia); Paolo BENANTI (Pontifical Gregorian University, Rome, Italy); Valentina BOETTO (Università degli studi di Torino); Paolo BOLDRINI (Società Italiana di Medicina Fisica e Riabilitativa, SIMFER); Donatella BONAIUTI (Istituto Geriatrico Piero Redaelli, Milan, Italy); Marco BRAVI (Biomedical Campus University Foundation, Rome, Italy); Federica BRESSI (Biomedical Campus University Foundation, Rome, Italy); Diletta BRUNO (Biomedical Campus University Foundation, Rome, Italy); Emanuela CASANOVA (IRCCS Istituto delle Scienze Neurologiche di Bologna, UOC Medicina Riabilitativa e Neuroriabilitazione, Bologna, Italia); Enrico CASTELLI (Paediatric Neurorehabilitation, Bambino Gesù Children's Hospital, Rome, Italy); Manuela DESILVESTRI (Neurorehabilitation Unit, Azienda Ospedaliera SS. Antonio e Biagio e Cesare Arrigo, Alessandria, Italy, EU); Francesco DRAICCHIO (Department of Occupational and Environmental Medicine, Epidemiology and Hygiene, INAIL, Monte Porzio Catone, 00185 Rome, Italy); Vincenzo FALABELLA (President Italian Federation of Persons with Spinal Cord Injuries (Faip Onlus), Rome, Italy); Serena FILONI (Padre Pio Foundation and Rehabilitation Centers, San Giovanni Rotondo, Italy); Silvia GALERI (IRCCS Fondazione Don Carlo Gnocchi, Milan, Italy); Marialuisa GANDOLFI (Department of Neurosciences, Biomedicine and Movement Sciences, University of Verona, Verona, Italy); Stefano GARGANO (Fondazione Don Carlo Gnocchi - Torino); Daniele GIANSANTI (National Center for Innovative Technologies in Public Health, Italian National Institute of Health, Rome, Italy); Francesca GIMIGLIANO (Department of Mental and Physical Health and Preventive Medicine, University of Campania "Luigi Vanvitelli", Naples, Italy); Mauro GRIGIONI (National Center for Innovative Technologies in Public Health, Italian National Institute of Health, Rome, Italy); Giuseppe LA ROSA (C.S.R. - Consorzio Siciliano di Riabilitazione, Catania, Italy); Lorenzo LIPPI (Università del Piemonte Orientale “A. Avogadro", Novara); Dario MARINO (IRCCS Neurolysis Center “Bonino Pulejo", Messina, Italy); Alex MARTINO CINNERA (Santa Lucia Foundation, IRCCS, Rome, Italy); Stefano MAZZOLENI (Department of Electrical and Information Engineering, Politecnico di Bari, Italy); Stefano MAZZON (AULSS6 (Unique Sanitary Local Company) Euganea Padova - Rehabilitation Department); Sandra MICCINILLI (Biomedical Campus University Foundation, Rome, Italy); Franco MOLTENI (Villa Beretta, Costa Masnaga, Italy); Giovanni MORONE (Santa Lucia Foundation, IRCCS, Rome, Italy); Matteo PACI (AUSL (Unique Sanitary Local Company) District of Central Tuscany, Florence, Italy); Angela PALOMBA (Multidisciplinary Department of Medicine for Surgery and Orthodontics, University of Campania "Luigi Vanvitelli", Naples, Italy); Cristiano PECCHIOLI (Fondazione Don Carlo Gnocchi, Roma); Luca PERRERO (Neurorehabilitation Unit, Azienda Ospedaliera SS. Antonio e Biagio e Cesare Arrigo, Alessandria, Italy, EU); Maurizio PETRARCA ("Bambino Gesù" Children's Hospital IRCCS, Movement Analysis and Robotics Laboratory MARlab, Rome, Italy); Salvatore PETROZZINO (A.O.U. Città della Salute e della Scienza di Torino); Alessandro PICELLI (Department of Neurosciences, Biomedicine and Movement Sciences, University of Verona, Verona, Italy); Federico POSTERARO (Versilia Hospital AUSL Toscana Nord Ovest, Italy); Emanuele Francesco RUSSO (Padre Pio Foundation and Rehabilitation Centers, San Giovanni Rotondo, Italy); Fabio SANTACATERINA (Biomedical Campus University Foundation, Rome, Italy); Michele SENATORE (Past President A.I.T.O. (Associazione Italiana Terapisti Occupazionali)); Monica SICARI (A.O.U. Città della Salute e della Scienza di Torino); Claudio Marcello SOLARO (CRRF 


\begin{abstract}
“Mons. Luigi Novarese” Moncrivello, VC, Italy); Silvia STERZI (Biomedical Campus University Foundation, Rome, Italy); Sofia STRAUDI (Ferrara University Hospital, Neuroscience and Rehabilitation Department, Ferrara, Italy); Giuseppe TURCHETTI (Management Institute, Sant'Anna School of Advanced Studies, Pisa, Italy).
\end{abstract}

\title{
References
}

1. Lu X, Battistuzzo CR, Zoghi M, Galea MP. Effects of training on upper limb function after cervical spinal cord injury: a systematic review. Clin Rehabil. 2015;29:3-13.

2. Stampas A, Tansey KE. Spinal cord injury medicine and rehabilitation. Semin Neurol. 2014;34:524-33.

3. Invernizzi M, de Sire A, Renò F, et al. Spinal Cord Injury as a Model of Bone-Muscle Interactions: Therapeutic Implications From in vitro and in vivo Studies. Front Endocrinol (Lausanne). 2020;11:204.

4. Löfvenmark I, Norrbrink C, Nilsson-Wikmar L, Hultling C, Chakandinakira S, Hasselberg M. Traumatic spinal cord injury in Botswana: characteristics, aetiology and mortality. Spinal Cord. 2015;53:150-154.

5. Pickett GE, Campos-Benitez M, Keller JL, Duggal N. Epidemiology of traumatic spinal cord injury in Canada. Spine (Phila Pa 1976). 2006;31:799-805.

6. Herrmann KH, Kirchberger I, Biering-Sorensen F, et al. Differences in functioning of individuals with tetraplegia and paraplegia according to the International Classification of Functioning, Disability and Health (ICF). Spinal Cord 2011;49:53443.

7. $\quad$ Spinal Cord Injury (SCI) Facts and Figures at a Glance. J Spinal Cord Med. 2016; 42016:493-4.

8. Brogioli M, Schneider S, Popp WL, et al. Monitoring upper limb recovery after cervical spinal cord injury: insights beyond assessment scores. Front Neurol; 2016;7:142.

9. Anderson KD. Targeting recovery: priorities of the spinal cord-injured population. J Neurotrauma 2004;21(10):1371-83.

10. French B, Thomas LH, Coupe J, et al. Repetitive task training for improving functional ability after stroke. Cochrane Database Syst Rev. 2016;11:CD006073.

11. Edgerton VR, Roy RR. Robotic training and spinal cord plasticity. Brain Res Bull. 2009;78:4-12.

12. Morone G, Paolucci S, Cherubini A, et al. Robot-assisted gait training for stroke patients: current state of the art and perspectives of robotics. Neuropsychiatr Dis Treat. 2017;13:1303-1311.

13. Riener R. Rehabilitation robotics. Foundations and Trends ${ }^{\circledR}$ in Robotics. 2013;3:1-137.

14. Morone G, Cocchi I, Paolucci S, Iosa M. Robot-assisted therapy for arm recovery for stroke patients: state of the art and clinical implication. Expert Rev Med Devices. 2020;17:223-233.

15. Turner DL, Ramos-Murguialday A, Birbaumer N, Hoffmann U, Luft A. Neurophysiology of robot-mediated training and therapy: a perspective for future use in clinical populations. Front Neurol. 2013;4:184.

16. Maciejasz P, Eschweiler J, Gerlach-Hahn K, Jansen-Troy A, Leonhardt, S. A survey on robotic devices for upper limb rehabilitation. J Neuroeng Rehabil. 2014;11:3.

17. Tramontano M, Morone G, Palomba A, De Angelis S, Mercuro A, Caltagirone C, Grasso MG. Effectiveness of a sensorbased technology in upper limb motor recovery in post-acute stroke neurorehabilitation: a randomized controlled trial. $J$ Biol Regul Homeost Agents. 2020;34:165-174. Technology in Medicine.

18. Tramontano M, Morone G, De Angelis S, Casagrande Conti L, Galeoto G, Grasso MG. Sensor-based technology for upper limb rehabilitation in patients with multiple sclerosis: A randomised controlled trial. Restor Neurol Neurosci. 2020;38:333341.

19. Morone G, Palomba A, Martino Cinnera A, et al.; "CICERONE" Italian Consensus Conference on Robotic in Neurorehabilitation. Systematic review of guidelines to identify recommendations for upper limb robotic rehabilitation after stroke. Eur J Phys Rehabil Med. 2021;57:238-245.

20. Gimigliano F, Palomba A, Arienti C, et al.; Italian Consensus Conference on Robotics in Neurorehabilitation (CICERONE). Robot-assisted arm therapy in neurological health conditions: rationale and methodology for the evidence synthesis in the CICERONE Italian Consensus Conference. Eur J Phys Rehabil Med. 2021 Jun 15.

21. Cooke A, Smith D, Booth A. Beyond PICO: the SPIDER tool for qualitative evidence synthesis. Qual Health Res. 2012;22:143543.

22. Moher D, Liberati A, Tetzlaff J, Altman DG; PRISMA Group. Preferred reporting items for systematic reviews and metaanalyses: the PRISMA statement. BMJ. 2009;339:b2535.

23. Shea BJ, Reeves BC, Wells G, Thuku M, Hamel C, Moran J, Moher D, Tugwell P, Welch V, Kristjansson E, Henry DA. AMSTAR 2: a critical appraisal tool for systematic reviews that include randomised or non-randomised studies of healthcare interventions, or both. BMJ. 2017;358:j4008.

24. Cashin AG and McAuley JH. Clinimetrics: physiotherapy evidence database (PEDro) scale. J Physiother 2020; 66(1): 59.

25. Zariffa J, Kapadia N, Kramer JL, et al. feasibility and efficacy of upper limb robotic rehabilitation in a subacute cervical spinal cord injury population. Spinal Cord. 2012;50:220-6.

26. Cortes M, Elder J, Rykman A, et al. Improved motor performance in chronic spinal cord injury following upper-limb robotic training. NeuroRehabilitation. 2013;33:57-65.

27. Fitle KD, Pehlivan AU, O'Malley MK. A Robotic Exoskeleton for rehabilitation and assessment of the upper limb following incomplete spinal cord injury. IEEE International Conference on Robotics and Automation, Seattle, May 26-30, 2015; 4960-6. 
28. Vanmulken DA, Spooren AI, Bongers HM, Seelen HA. Robot-assisted task-oriented upper extremity skill training in cervical spinal cord injury: a feasibility study. Spinal Cord. 2015;53:547-51.

29. Francisco GE, Yozbatiran N, Berliner J, O’Malley MK, Pehlivan AU, Kadivar Z, Fitle K, Boake C. Robot-Assisted Training of Arm and Hand Movement Shows Functional Improvements for Incomplete Cervical Spinal Cord Injury. Am J Phys Med Rehabil. 2017;96:S171-S177.

30. Frullo JM, Elinger J, Pehlivan AU, et al. Effects of Assist-As-Needed Upper Extremity Robotic Therapy after Incomplete Spinal Cord Injury: A Parallel-Group Controlled Trial. Front Neurorobot. 2017;11:26.

31. Kim J, Lee BS, Lee HJ, et al. Clinical efficacy of upper limb robotic therapy in people with tetraplegia: a pilot randomised controlled trial. Spinal Cord. 2019;57:49-57.

32. Singh H, Unger J, Zariffa J, Pakosh M, Jaglal S, Craven BC, Musselman KE. Robot-assisted upper extremity rehabilitation for cervical spinal cord injuries: a systematic scoping review. Disabil Rehabil Assist Technol. 2018;13:704-715.

33. Yozbatiran N, Francisco GE. Robot-assisted Therapy for the Upper Limb after Cervical Spinal Cord Injury. Phys Med Rehabil Clin N Am. 2019;30:367-384.

34. Jung JH, Lee HJ, Cho DY, et al. Effects of Combined Upper Limb Robotic Therapy in Patients With Tetraplegic Spinal Cord Injury. Ann Rehabil Med. 2019;43:445-457.

35. Osuagwu BAC, Timms S, Peachment R, et al. Home-based rehabilitation using a soft robotic hand glove device leads to improvement in hand function in people with chronic spinal cord injury:a pilot study. J Neuroeng Rehabil. 2020;17:40.

36. OCEBM Levels of Evidence - Centre for Evidence-Based Medicine, University of Oxford. https://www.cebm.ox.ac.uk/resources/levels-of-evidence/ocebm-levels-of-evidence (accessed February 13, 2021).

37. Carpinella I, Cattaneo D, Abuarqub S, Ferrarin M. Robot-based rehabilitation of the upper limbs in multiple sclerosis: feasibility and preliminary results. J Rehabil Med. 2009;41:966-70.

38. Vanoglio F, Bernocchi P, Mulè C, Garofali F, Mora C, Taveggia G, Scalvini S, Luisa A. Feasibility and efficacy of a robotic device for hand rehabilitation in hemiplegic stroke patients: a randomised pilot controlled study. Clin Rehabil. 2017;31:351360.

39. Yozbatiran N, Keser Z, Davis M, et al. Transcranial direct current stimulation (tDCS) of the primary motor cortex and robotassisted arm training in chronic incomplete cervical spinal cord injury: A proof of concept sham-randomised clinical study. NeuroRehabilitation. 2016;39:401-11.

40. Pehlivan AU, Celik O, O'Malley MK. Mechanical design of a distal arm exoskeleton for stroke and spinal cord injury rehabilitation. IEEE Int Conf Rehabil Robot. 2011;2011:5975428 